\title{
Der Zinssatz ist kein Preis
}

\author{
Die Interpretation des Zinssatzes als Preis ist problematisch. Im Gegensatz zum Preis ist der \\ Zinssatz kein Austauschverhältnis. Modelle, in denen der Zinssatz mit einem Preis für die \\ Nutzung von Kapital identifiziert werden kann, sind sehr speziell. In allgemeineren Modellen \\ sind Zinssatz und Mietpreise für Kapital verschieden. Die dogmengeschichtliche Entwicklung \\ hinter dieser Diskussion deutet auf eine apologetische Funktion der Interpretation des \\ Zinssatzes als Mietpreis für Kapital. Im Hintergrund steht die Frage, in welchen Einheiten \\ Zinssatz und „Kapital“ gemessen werden.
}

\begin{abstract}
Für Carl Christian von Weizsäcker, der mir für die Diplomarbeit 1970 die Frage stellte: "Ist der Zins als realwirtschaftliches oder als monetäres Phänomen zu interpretieren?“
\end{abstract}

Vor einiger Zeit las ich in einer prominenten deutschen Zeitung sinngemäß: Der Zinssatz ist der Preis auf dem Geldmarkt. Preise sind positiv. Wenn der Zinssatz heute negativ ist, muss das daran liegen, dass die Politik der EZB das Marktgeschehen verfälscht. Als ich das las, dachte ich, da hat jemand die Entwicklungen der Wirtschaftstheorie im dritten Quartal des 20. Jahrhunderts vergessen.

Das war allerdings kein Einzelfall. Als ich kürzlich in einem Zeitungsartikel (Hellwig, 2021a) schrieb „Der Zinssatz ist kein Preis", äußerten zwei Korrespondenten, der Zinssatz sei doch der Preis für die Nutzung von Kapital. Ein Jurist verwies mich auf den Münchner Kommentar zu § 488 BGB, wo diese Sicht als "herrschende Meinung" dargestellt wird. Ein Ökonom meinte, das stehe schon bei Wicksell, dem schwedischen Ökonomen, mit dessen Buch Geldzins und Güterpreise (1898) die moderne Geldtheorie inren Anfang nahm. Diese Interpretation des Zinssatzes ist allerdings problematisch.

(C) Der/die Autor:in 2021. Open Access: Dieser Artikel wird unter der Creative Commons Namensnennung 4.0 International Lizenz veröffentlicht (creativecommons.org/licenses/by/4.0/deed.de).

Open Access wird durch die ZBW - Leibniz-Informationszentrum Wirtschaft gefördert.

Prof. Dr. Martin Hellwig ist emeritierter Direktor am Max-Planck-Institut zur Erforschung von Gemeinschaftsgütern in Bonn.

\section{Der Zinssatz ist kein Austauschverhältnis}

Ein Preis ist ein Austauschverhältnis. Ein Preis gibt an, wie viele Einheiten von einem Objekt man hergeben muss, um eine Einheit eines anderen Objekts zu bekommen, z.B. wie viele runde Metallstückchen mit der Prägung „1 Euro“ man hergeben muss, um das von Anat Admati und mir geschriebene Buch „Des Bankers neue Kleider" zu bekommen.

Der Zinssatz ist kein Austauschverhältnis. Wenn ich heute eine Festgeldanlage über 1.000 Euro zu einem Zinssatz von $0,5 \%$ über zwölf Monate tätige, so bekomme ich in einem Jahr (hoffentlich) eine Auszahlung von $1.005 \mathrm{Eu}$ ro. Das Austauschverhältnis zwischen der Auszahlung in einem Jahr und der Einzahlung heute beträgt 1005:1000 $=1,005$. Wäre der Zinssatz negativ, z.B. minus $0,5 \%$, so bekäme ich nach einem Jahr nur 995 Euro; das Austauschverhältnis zwischen der Auszahlung in einem Jahr und der Einzahlung heute läge bei 0,995. Obwohl der Zinssatz negativ ist, wäre das Austauschverhältnis positiv. Das Austauschverhältnis zwischen Geld, das in einem Jahr zur Verfügung steht, und Geld, das ich jetzt in der Hand habe, hängt vom Zinssatz ab, ist aber nicht mit dem Zinssatz gleichzusetzen.

Gewiss kann man die Zahlung, die ich in einem Jahr erhalte, gedanklich in eine Rückzahlung von 1.000 Euro und eine Zinszahlung von 5 Euro aufteilen. Das ist aber nur ein gedankliches Konstrukt. Dem einzelnen Euro, den ich in einem Jahr bekomme, werde ich nicht ansehen, ob er zur Rückzahlung oder zu den Zinsen gehört.

Der Punkt wird noch deutlicher, wenn ich statt der genannten Festgeldanlage eine Anleihe mit einem Kuponzins von $5 \%$ und einem Jahr Restlaufzeit betrachte. Beträgt der Nennwert der Anleihe 1.000 Euro, bekomme ich in einem Jahr 1.050 Euro. Liegt der 12-Monats-Marktzins bei $0,5 \%$, so muss ich dafür allerdings ca. 1.045 Euro be- 
zahlen, sodass die Rendite über zwölf Monate genau dem 12-Monats-Marktzins entspricht. Bei dieser Transaktion ist der heutige Verkäufer der Anleihe nicht der Schuldner der Anleihe, und die Rendite von 0,5\%, die ich bekomme, hat nichts mit dem Vertrag zu tun, unter dem die Anleihe ausgegeben wurde. Aus Sicht des Schuldners der Anleihe besteht die Zahlung von 1.050 Euro in einem Jahr aus einer Rückzahlung von 1.000 Euro und einer Zinszahlung von 50 Euro, aus meiner Sicht aus dem Mitteleinsatz von 1.045 Euro und einer Verzinsung von 5 Euro.

Was der Verkäufer mit dem Erlös der Anleihe macht, ob er inn für ein opulentes Essen verwendet oder ob er dieses Kapital „für sich arbeiten lässt“, ist mir egal und geht mich auch nichts an, denn ich habe weiter keinen Anspruch gegen ihn. Die Relevanz der Rendite von 0,5\% für diese Transaktion ergibt sich aus dem Prinzip der Einheitlichkeit des Preises (des Austauschverhältnisses) über alle Arten von Tausch von Geld jetzt gegen Geld, das in einem Jahr zur Verfügung steht, bei denen die Zahlung in einem Jahr als sicher angesehen wird.

\section{Oder doch? Der Zinssatz in der naiven}

Grenzproduktivitätstheorie der Verteilung

Jedoch wird in einem einflusseichen makroökonomischen Modell der Preis für die Nutzung von Kapital als Zinssatz bezeichnet (zum Folgenden siehe Bliss, 1975). In diesem Modell produziert ein Unternehmen einen Output $y$ mit Inputs $\ell$ und $k$ an Arbeit und Kapital gemäß einer Produktionsfunktion

$$
y=f(k, \ell)
$$

Das Unternehmen wählt einen Produktionsplan $(y, K, L)$, um seinen Gewinn

$$
y-R k-w \ell
$$

zu maximieren. Dabei ist $R$ der Preis, zu dem es Kapital von den Kapitaleigner:innen mietet, und $w$ der Lohnsatz. Für die Gewinnmaximierung müssen $\mathrm{k}$ und $\ell$ so gewählt werden, dass die Bedingungen

$$
R=\frac{\partial f(k, \ell)}{\partial k} \text { und } w=\frac{\partial f(k, \ell)}{\partial \ell}
$$

erfüllt sind, d.h. die Grenzprodukte von Kapital und Arbeit, die Erträge der letzten eingesetzten Einheiten an Kapital und Arbeit, müssen gleich dem Mietpreis $R$ und dem Lohnsatz w sein.

Zu einem gegebenen Zeitpunkt sind die insgesamt verfügbaren Mengen $L, K$ an insgesamt verfügbarer Arbeit und verfügbarem Kapital vorgegeben. Ein Marktgleichgewicht ergibt sich, wenn die Inputpreise R und w sich so anpassen, dass die Gesamtnachfrage der Unternehmen nach diesen Inputs gerade diesen Mengen entspricht. Im einfachsten Falle wählen alle Unternehmen denselben Produktionsplan. Interpretiert man $L$ und $K$ als verfügbare Mengen pro Unternehmen, so lauten die Markträumungsbedingungen

$$
k=K \quad \text { und } \quad \ell=L \text {, }
$$

wobei $k$ und $\ell$ die gewählten Inputs sind. In einem solchen Gleichgewicht sind daher der Kapitalmietpreis R und der Lohnsatz w auch gleich den Grenzprodukten von Kapital und der Arbeit bei dem Inputeinsatz K, L. Weist die Produktionsfunktion konstante Skalenerträge auf, so folgt

$$
y=R K+w L
$$

d.h. die gesamte Wertschöpfung wird für die Bezahlung der Inputs gebraucht. Die Unternehmensgewinne sind Null.

Dieses Modell enthält den Kern der Grenzproduktivitätstheorie der funktionalen Einkommensverteilung. In der hier skizzierten naiven Form hängen Arbeitseinkommen und Kapitaleinkommen nur von den verfügbaren Mengen an Arbeit und Kapital und von der Produktionsfunktion ab. „Kapital“ ist dabei als eine physische Größe zu verstehen, als Maß für die Maschinen und Ähnliches, welche die Unternehmen bei der Produktion einsetzen.

In diesem Modell liegen Eigentum und Nutzung des Kapitals in verschiedenen Händen, anders als zumeist in der Realität. ${ }^{1}$ Diese Trennung von Eigentum und Nutzung ist die Grundlage dafür, dass es einen Markt für die Nutzung von Kapitalgütern gibt - und einen Preis für diese Nutzung.

Diesen Preis als „Zinssatz" zu bezeichnen, ist insofern unschädlich, als im Modell die Märkte gar nicht vorkommen, auf denen Zinssätze in der Realität eine Rolle spielen. Die Finanzierung des Erwerbs von Kapitalgütern wird nicht behandelt. Die Interpretation des Zinssatzes als eines Preises für die Nutzung von Kapital beruht letztlich darauf, dass das Modell die Märkte, auf denen in der Realität Zinssätze eine Rolle spielen, ausschließt, dafür aber einen in der Realität kaum vorkommenden Markt einführt und den auf diesem Markt geltenden Preis als "Zinssatz“ bezeichnet. Es fragt sich, ob das mehr ist als ein Spiel mit der Semantik des Wortes „Zinssatz“.

1 Die bei Aktiengesellschaften hin und wieder gebräuchliche Forme von Aktionären als Eigentümern des Unternehmens und seiner Vermögenswerte ist irreführend. Die AG als juristische Person ist Eigentümerin der Maschinen und anderer Vermögenswerte, die Aktionäre sind Eigentümer von Aktien und haben keine Verfügungsrechte über die Vermögenswerte der Aktiengesellschaft. 


\section{Mietpreise sind keine Zinssätze}

Im Modell der naiven Grenzproduktivitätstheorie gibt es nur ein produziertes Gut. Beim Output des Unternehmens - oder der Volkswirtschaft - wird nicht unterschieden, ob dieser Output für Konsum oder für Investitionen verwendet wird. Daher spielen Output-Preise keine Rolle. Ein Tausch Kapitalgut gegen Konsumgut findet nicht statt, wenn jede Einheit Output gleichermaßen als Konsumgut und als Kapitalgut verwendet werden kann.

Was ergibt sich, wenn man mehr als ein Gut zulässt? Im einfachsten Fall gibt es ein Konsumgut und ein Kapitalgut. In diesem Fall wählt ein Konsumgutproduzent einen Produktionsplan $\left(y_{c}, k_{c}, \ell_{c}\right)$, um den Gewinn

$$
p_{c} y_{c}-R k_{c}-w \ell_{c}=p_{c} f_{c}\left(k_{c}, \ell_{c}\right)-R k_{c}-w \ell_{c}
$$

zu maximieren, ein Kapitalgutproduzent einen Produktionsplan $\left(y_{k}, k_{k}, \ell_{k}\right)$, um den Gewinn

$$
p_{k} y_{k}-R k_{k}-w \ell_{k}=p_{k} f_{k}\left(k_{k}, \ell_{k}\right)-R k_{k}-w \ell_{k}
$$

zu maximieren. Dabei sind $p_{c}$ und $p_{k}$ die Preise, zu denen die Unternehmer:innen ihre jeweiligen Outputs nach Fertigstellung zu verkaufen gedenken; $R$ und $w$ sind wieder der Mietpreis pro Einheit des Kapitalguts und der Lohnsatz. Gewinnmaximierung erfordert nunmehr

$$
R=p_{c} \frac{\partial f_{c}\left(k_{c}, \ell_{c}\right)}{\partial k_{c}} \text { und } p_{k} \frac{\partial f_{k}\left(k_{k}, \ell_{k}\right)}{\partial k_{k}}
$$

und

$$
w=p_{c} \frac{\partial f_{c}\left(k_{c}, \ell_{c}\right)}{\partial \ell} \text { und } p_{k} \frac{\partial f_{k}\left(k_{k}, \ell_{k}\right)}{\partial \ell} \text {. }
$$

Der Mietpreis $R$ pro Einheit Kapital muss gleich dem Marktwert des Grenzprodukts des Kapitals in jeder Verwendung sein.

Die Aufteilung der Ressourcen Arbeit und Kapital auf die beiden Verwendungsrichtungen hängt von den Outputpreisen $p_{c}$ und $p_{k}$ ab und diese wiederum von der Nachfrage nach Konsumgütern und neuen Kapitalgütern durch private Haushalte und Unternehmen bzw. Investierende. Im Unterschied zur naiven Grenzproduktivitätstheorie der Verteilung sind die Inputpreise $R$ und $w$ hier nicht mehr rein technisch bestimmt; sie hängen auch von der Nachfrageseite der Volkswirtschaft ab. Die Preise $p_{c}$ und $p_{k}$ koordinieren Nachfrage und Produktion.

Darf man R als Zinssatz bezeichnen? Bei oberflächlicher Betrachtung könnte die Antwort auf diese Frage davon abhängen, in welcher Einheit $R$ definiert ist. Definieren wir $\mathrm{R}$ als Zahl von Einheiten des Konsumguts, die je eingesetzter Einheit des Kapitalguts zu bezahlen sind, dann hat $\mathrm{R}$ die Dimension Einheiten des Konsumguts je eingesetzter Einheit des Kapitalguts. Dagegen ist ein Zinssatz dimensionslos, eine natürliche Zahl, z. B. $5 \%=0,05$. Insofern kann R nicht als Zinssatz interpretiert werden.

Definiert man R stattdessen als die Zahl von Einheiten des Kapitalguts, die je eingesetzter Einheit des Kapitalguts zu bezahlen sind, so ist R dimensionslos und könnte als Zinssatz interpretiert werden. Das entspräche der Konstellation im obigen Modell mit nur einem Gut, bei dem der Mietpreis dimensionslos ist, wenn Output und Kapitalinput dasselbe Gut sind.

Diese Interpretation enthält aber ein Element der Willkür. Das wird deutlich, wenn es noch ein zweites Kapitalgut gibt, das vom ersten verschieden ist. Dann ist zwischen den Mietpreisen $R_{1}$ und $R_{2}$ für das erste und das zweite Kapitalgut zu unterscheiden. Die geforderten Leistungen müssen bei beiden in denselben Einheiten spezifiziert sein, denn sonst könnte man nicht die Summe $R_{1}$ $k_{1}+R_{2} k_{2}$ für die Kapitalkosten insgesamt bilden. Nimmt man das erste Kapitalgut als Wertmaßstab und definiert $R_{1}$ und $R_{2}$ als Zahl von Einheiten dieses Kapitalguts, die je eingesetzter Einheit von Kapitalgut 1 bzw. Kapitalgut 2 als Miete zu bezahlen sind, dann ist $R_{2}$ nicht dimensionslos und kann nicht als Zinssatz interpretiert werden. Nimmt man umgekehrt das zweite Kapitalgut als Wertmaßstab, dann ist $R_{1}$ nicht dimensionslos und kann nicht als Zinssatz interpretiert werden. Die Wahl der Werteinheit ist aber willkürlich.

\section{Eigentliche Funktion von Ertragsrate und Zinssatz}

Der Versuch, den Zinssatz als Mietpreis für Kapital bzw. für Kapitalgüter zu interpretieren (oder umgekehrt), lenkt von der eigentlichen Funktion des Zinssatzes ab, der Lenkung von Investitionsmitteln. Zwei Lenkungsprobleme sind zu unterscheiden.

Das eine betrifft die Zuordnung der verfügbaren Kapitalgüter zu den einzelnen Produktionsaktivitäten. Hier sind die Mietpreise der Kapitalgüter wichtig. Bei einem einheitlichen Mietpreis $R_{j}$ für Kapitalgut $j$ sind die Eigentümer:innen dieses Kapitalguts indifferent, welchem Produzenten sie das Kapitalgut vermieten. Es kommt nur darauf an, ob ein Produzent bereit ist, den Mietpreis $R_{j}$ zu bezahlen. Ob und in welchem Ausmaß das der Fall ist, hängt von den Gewinnmöglichkeiten der Produzenten ab. Die Mietpreise selbst müssen sich so anpassen, dass für jedes einzelne Kapitalgut die Nachfrage der Unternehmer:innen insgesamt gerade den verfügbaren Bestand an diesem Kapital abdeckt. 
Das zweite Lenkungsproblem betrifft die Frage, wie die für Investitionen verfügbaren Mittel auf die verschiedenen Typen von Kapitalgütern aufgeteilt werden. Diese Frage wurde bisher ausgeklammert. Sie ist auch uninteressant, wenn die Bestände der Kapitalgüter als vorgegeben angenommen werden. Zu einem gegebenen Zeitpunkt mögen sie das sein, aber es ist auch wichtig, wie es zu diesen Beständen gekommen ist und wie sich Investitionen und Kapitalbestände im Zeitablauf entwickeln.

Der Investor, der Mittel einsetzt, um Kapitalgüter zu erwerben, wird erwägen, dass Kapitalgut $j$ pro Einheit eine Miete $R_{j}$ erbringt, beim Erwerb aber $q_{j}$ kostet. Er wird auch erwägen, dass das Kapitalgut unter Berücksichtigung des Kapitalverzehrs durch Abnutzung hinterher pro Einheit noch einen Restwert $p_{j}(1-\delta)$ haben wird; hier ist $\delta$ ein Maß für die Abnutzung je Einheit. ${ }^{2}$

Der Bruttoertrag je Einheit von Kapitalgut $j$ beläuft sich auf $R_{j}+p_{j}(1-\delta)$. Die Bruttoertragsrate je Einheit der eingesetzten Mittel beträgt demnach $\left(R_{j}+p_{j}(1-\delta)\right) / q_{j}$. Will der Investor die Erträge auf die eingesetzten Mittel maximieren, so investiert er nur dann in Kapitalgut $j$, wenn es kein anderes Kapitalgut gibt, bei dem die Ertragsrate höher ist. Alle Kapitalgüter, in die investiert wird, müssen daher dieselbe Ertragsrate aufweisen. Es gibt daher eine Zahl $\rho$, sodass

$$
(1+\rho) q_{j}=R_{j}+p_{j}(1-\delta)
$$

für alle Kapitalgüter $j$, in die investiert wird, und

$$
(1+\rho) q_{j} \geq R_{j}+p_{j}(1-\delta)
$$

für alle Kapitalgüter $j$, in die nicht investiert wird. Die Zahl $\rho$ gibt die gemeinsame Nettoertragsrate auf die Investitionen an, die getätigt werden.

Mietpreis und Nettoertragsrate, $R_{j}$ und $\rho$, sind zwei verschiedene Dinge. Der Mietpreis ist definiert in Einheiten des Wertmaßstabs je Einheit des Kapitalguts und wird von Kapitalgut zu Kapitalgut verschieden sein. Die Nettoertragsrate ist spezifiziert in Einheiten des Wertmaßstabs je eingesetzter Einheit des Wertmaßstabs, z.B., wenn man das Konsumgut als Wertmaßstab nimmt, in Einheiten des Konsumguts je Einheit Konsumverzicht und ist für alle Kapitalgüter gleich. Der Mietpreis ist ein Austauschverhältnis, desgleichen der Kapitalgutpreis $q_{k}$. Die Ertragsrate dagegen ist kein Austauschverhältnis.

2 Der Erwerbspreis $q_{j}$ ist grundsätzlich zu unterscheiden vom Preis $p_{j}$, zu dem ein Unternehmer als Nutzer des Kapitals seine neue Produktion des Kapitalguts verkauft. Der eine Preis bezieht sich auf den Zeitpunkt vor der Produktion, der andere auf den Preis nach der Produktion. Die beiden Preise können übereinstimmen, müssen es aber nicht.
Unterstellt man, dass der Investor sich seine Mittel erst beschaffen muss, indem er einen Kredit zu einem Zinssatz $r$ aufnimmt, so wird im Gleichgewicht die Nettoertragsrate $\rho$ auf Kapitalgüterinvestitionen mit dem Zinssatz $r$ übereinstimmen müssen. Wäre $\rho$ kleiner als $r$, so würde sich das Ganze für den Investor nicht lohnen; wäre $\rho$ größer als $r$, so würde er seine Mittelaufnahme unbegrenzt ausbauen und mehr Mittel aufnehmen wollen, als der Markt hergeben kann.

Im Gleichgewicht gilt daher

$$
(1+r) q_{j}=R_{j}+p_{j}(1-\delta)
$$

für alle Kapitalgüter $j$, in die investiert wird, und

$$
(1+r) q_{j} \geq R_{j}+p_{j}(1-\delta)
$$

für alle Kapitalgüter $j$, in die nicht investiert wird. Der Zinssatz $r$ liefert den Maßstab für die Ertragsrate, die ein Kapitalgut aufweisen muss, damit Mittel dafür aufgewandt werden. Dabei sind Zinssatz und Kapitalmietpreise verschiedene Dinge. Eine Gleichheit von Zinssatz und Mietpreis eines Kapitalguts $j$ ergibt sich nur, wenn $\delta=0$ sowie $q_{j}=p_{j}=1$. Diese Bedingungen sind sehr speziell.

Bei dem Wort „Kapital“ steht immer die Frage im Raum, ob es sich um eine Mengengröße oder um eine WertgröBe handelt. " „Kapital" als Input in die Produktion ist eine Mengengröße. Da der Mietpreis sich auf diese Mengengröße bezieht, ist er normalerweise nicht dimensionslos. Ertragsraten und Zinssätze dagegen beziehen sich auf Wertgrößen. Die Unterscheidung von „Kapital“ als Mengengröße und als Wertgröße entfällt nur im Spezialfall des Modells mit nur einem Gut, das ebenso gut als Konsumgut und als Kapitalgut dienen kann, sodass der Preis des Kapitalguts in Einheiten des Wertmaßstabs immer Eins ist.

Die Überlegung zur Lenkungsfunktion des Zinssatzes gilt auch, wenn man auf die Kunstfigur des Kapitalvermieters verzichtet und den Unternehmer unmittelbar Mittel zur Finanzierung von Kapitalgütern aufnehmen lässt. In diesem Fall ist der Gewinn aus der Produktion eines Gutes i gegeben als

$$
p_{i} f_{i}\left(k_{i 1}, \ldots, k_{i J}, \ell_{i}\right)+\Sigma_{j} p_{j}(1-\delta) k_{i j}-\Sigma_{j}(1+r) q_{j} k_{i j}-w \ell_{k}
$$

3 Eng damit verbunden ist die Frage, ob „Kapital“ als Vermögenswert auf der Aktivseite der Bilanz steht oder als Maß für die durch verschiedene Finanzierungen erhaltenen Mittel auf der Passivseite der Bilanz. Die Formulierung von der Nutzung des „Kapitals“ des Gläubigers durch den Schuldner bringt diese beiden Bedeutungen des Worts durcheinander: Der Gläubiger nutzt sein „Kapital“ (Wertgröße auf der Passivseite der Bilanz), indem er dem Schuldner Geld gibt (WertgröBe auf der Aktivseite der Bilanz), mit dem dieser Kapitalgüter kaufen kann; für den Nutzen, den dieser daraus zieht, kommt es auf die Mengen dieser Kapitalgüter an. 
und die Gewinnmaximierungsbedingung für den Einsatz des Kapitalguts $j$ durch diesen Produzenten lautet

$$
(1+r) q_{j}=p_{i} \frac{\partial f_{i}}{\partial k_{i j}}+p_{j}(1-\delta),
$$

im Einklang mit den vorherigen Ergebnissen.

\section{Kann der Gleichgewichtszinssatz negativ sein?}

Wenn die Rate des Kapitalverzehrs durch Abnutzung positiv ist, ist nicht auszuschließen, dass der Gleichgewichtszinssatz negativ ist. Dies gilt auch im naiven Modell mit nur einem Gut, das gleichzeitig Konsumgut und Kapitalgut ist. In diesem Modell, mit Notation $R$ für die Kapitalmiete und $r$ für den Kreditzinssatz, mit Güterpreisen $q=p=1$, findet man

$$
1+r=1+\rho=R+1-\delta
$$

und daher

$$
r=\frac{\partial f(K, L)}{\partial k}-\delta
$$

Wenn $K$ sehr groß ist in Relation zu $L$, ist das Grenzprodukt $\partial f(K, L) / \partial k$ möglicherweise kleiner als der Kapitalverzehrkoeffizient $\delta$. Dann ist die Nettoertragsrate auf eine Erhöhung von $\mathrm{K}$ negativ und damit auch der Zinssatz $r$.

Kann das in einem Gleichgewicht vorkommen? Die Antwort auf diese Frage hängt vom Verhalten der Menschen ab, die Kapital erwerben und halten. Denken wir z. B. an Personen, die sparen, um Vorsorge für ihre Altersversorgung zu treffen, sei es individuell, sei es durch eine Rentenversicherung mit Kapitaldeckung. Wenn dies viele tun, so kann das Angebot an Sparmitteln sehr groß sein und das auch, wenn die Nettoertragsrate auf diese Ersparnis negativ ist. Wenn privates Sparen die einzige Möglichkeit ist, dem Verhungern im Alter zu entgehen, dann nimmt man auch in Kauf, dass man im Alter weniger herausbekommt, als man angelegt hat. ${ }^{4}$ Wenn gleichzeitig die nächste Generation, die mit dem Kapital arbeiten wird, sehr klein ist, dann hat man eine Situation, die einer Modellkonstellation mit hohem $K$ und niedrigem $L$ entspricht. ${ }^{5}$

4 Wenn anders als im Modell auch Bargeld als Wertaufbewahrungsmittel zur Verfügung steht, kann der Nominalzins nicht deutlich negativ sein, wohl aber der Realzins.

5 Böhm-Bawerk (1884), gab drei Gründe an, warum der Gleichgewichtszins positiv sein könnte: 1 . Anleger ziehen bei konstanten Einkommen früheren Konsum gegenüber späterem Konsum vor; 2. Anleger rechnen mit steigenden Einkommen; 3. „Produktionsumwege“ sind produktiv, wobei Letzteres die österreichische Variante der Produktivität von Kapital ist. Böhm-Bawerks zweiter Grund wirkt mit umgekehrtem Vorzeichen, wenn Leute im Alter kein Arbeitseinkommen haben.
Den Vertrag zwischen den Sparenden und denen, die die gesparten Mittel nutzen, kann man auch als Verwahrungsvertrag interpretieren. Wenn den Anleger:innen sehr viel daran liegt, Kaufkraft in die Zukunft zu transferieren, so müssen sie möglicherweise einen Preis zahlen für die Verwahrung ihrer Sparmittel. Ein negativer „Zins“ entspricht einem solchen Preis für die Verwahrung von Mitteln.

Ob der Gleichgewichtszins positiv oder negativ ist und ob wir es vorrangig mit einem Vertrag über die Nutzung von Mitteln oder einem Vertag über die Verwahrung von Mitteln zu tun haben, hängt von den Parametern des Modells ab, von der Produktionsfunktion, der Abschreibungsrate und dem Bedarf der Anleger nach einem Transfer von Kaufkraft in die Zukunft (ausführlich Weizsäcker und Krämer, 2019).

Die Frage Verwahrung oder Nutzung ist Gegenstand einer Kontroverse zu den Ursprüngen des englischen Banksystems im 17. Jahrhundert. Reiche Leute in England hatten die Gewohnheit, Gold im Tower aufbewahren zu lassen. Im Bürgerkrieg konfiszierte der König dieses Gold. Danach ging man dazu über, Goldbestände bei Goldschmieden zur Verwahrung zu geben. Die Goldschmiede merkten bald, dass es sich um eine Speziesschuld handelte und dass nie alle Bestände auf einmal abgezogen wurden, und sie fingen an, „überschüssige“ Goldreserven zur Kreditvergabe an Dritte zu verwenden.

Die Frage ist, ob dieses Verhalten als Vertragsbruch anzusehen ist oder nicht. Für die Interpretation als Vertragsbruch wird angeführt, dass es sich um Verwahrungsverträge handelte, wie beim Gold im Tower oder bei der Gepäckaufbewahrung am Bahnhof (Rothbard, 2008). Gegen die Interpretation als Vertragsbruch wird angeführt, dass die Goldschmiede alsbald positive Zinsen auf Goldeinlagen boten, und dass die Einleger wussten oder wissen mussten, warum das geschah (Selgin, 2012).

Für den Transfer von Kaufkraft über die Zeit kommen auch Vermögenswerte wie Immobilien oder Geld infrage. Wenn deren Preise hinreichend groß sind, können diese Vermögenswerte jeglichen Bedarf nach Wertaufbewahrung befriedigen. Aufgrund dieser Erwägung vertritt etwa Homburg (2014) die Auffassung, in der Realität könnte der Gleichgewichtszins nie negativ sein (auch Sinn, 2020). Bei Land als Wertaufbewahrungsmittel (Hellwig, 2021b) ist diese Aussage allerdings nicht robust gegenüber der Einführung von Transaktionskosten und von Unsicherheit (Weizsäcker und Krämer, 2019; Hellwig, 2021c). Bei Geld steht die Frage im Raum, ob die bei einem Anstieg in der Nachfrage nach Wertaufbewahrungsmitteln erforderliche Wertsteigerung des Geldes, d.h. Deflation, reibungslos erfolgen kann. 


\section{Historische Hintergründe der Diskussion}

Wo kommt das naive Modell eigentlich her? Um diese Frage zu beantworten, skizziere ich die historische Entwicklung der Zinstheorie. Ich stütze mich dabei auf Joseph Schumpeters History of Economic Analysis (1954). Nach Schumpeter beginnt die Zinstheorie bei den Scholastikern, in der Auseinandersetzung mit dem kirchlichen Zinsverbot. Für Thomas von Aquin war Zins Wucher, da ihm keine Leistung gegenüberstand (Schumpeter, 1954, 94). ${ }^{6}$ Demgegenüber verweisen spätere Scholastiker auf die Gewinne, welche die Kreditnehmenden, vor allem Kaufleute, aus der Nutzung von Geld als „Merchants“ Tool“ ziehen (Schumpeter, 1954, 104 ff.). Das Spannungsverhältnis zwischen kirchlichem Zinsverbot und empirischer Praxis wird dadurch aufgelöst, dass man die Kreditvergabe nicht mit Notsituationen von Privatpersonen, sondern mit Ertragspotenzialen von Geschäftsleuten in Verbindung bringt, die die von Gläubigern beigesteuerten Mittel zur Ausweitung ihrer Aktivitäten verwenden. Allerdings fehlt eine Analyse des Zusammenhangs zwischen den Ertragspotenzialen der Geschäftsleute und den Zinsen auf die aufgenommenen Mittel.

Die englischen Ökonomen des späten 17. und des 18. Jahrhunderts „lösen“ dieses Problem, indem sie die Unterscheidung zwischen Unternehmensgewinnen und Zinsen beiseiteschieben und beides zusammen als Einkommen auf Kapital (Stock) bezeichnen (Schumpeter, 1954, 329 ff.). Der Pionier ist Nicholas Barbon (A Discourse of Trade, 1690): „Interest is commonly reckoned for Money... but this is a mistake; for Interest is paid for Stock; it is the Rent of Stock, and is the same as the Rent of Land; the First is Rent of Wrought or Artificial Stock; the Latter, of the Unwrought or Natural Stock" (zitiert nach Schumpeter, 1954). Diese Idee wird von den Klassikern Adam Smith und David Ricardo übernommen. Diese unterscheiden nur Löhne, Grundrenten und Profite und subsumieren Zinseinkommen unter Profiten. Dabei setzen sie das Wort "capital“ an die Stelle des von Barbon verwendeten Wortes "Stock“" ${ }^{7}$

Dabei gerät der Zinssatz aus dem Blickfeld. Stattdessen spricht man über die „Profitrate“, das Verhältnis der Profite zum eingesetzten Kapital, entsprechend der Größe $\rho$

6 Thomas von Aquin verwirft das Argument, der Zins sei ein Preis für die Nutzung von Geld, mit dem Hinweis darauf, dass aus Sicht des Verleihers das Geld durch die Kreditvergabe verbraucht werde, es mithin keine Nutzung gebe, die von der Substanz des Geldes getrennt werden könnte; ein Preis für etwas, das es nicht gebe, sei abzulehnen.

7 Schumpeter (1954) weist auf diesen Wandel des Sprachgebrauchs hin. Dabei geht es auch um die Frage, ob „Kapital“ die Passivseite der Bilanz betrifft (wie in „Eigenkapitalanforderungen für Banken“) oder die Aktivseite der Bilanz (wie in „Kapitalstock“). Zur Ambivalenz und zur Entwicklung des Sprachgebrauchs siehe auch Braudel, 1990, Kap. 3. in der obigen Analyse. Ausgangspunkt ist die empirische Beobachtung, dass Unternehmer:innen und Anlegende ihre Mittel dort einsetzen, wo sich die höchsten Profitraten erzielen lassen, und dass dieses Verhalten tendenziell zu einer Angleichung der Profitraten in den verschiedenen Einsatzmöglichkeiten führt. Die Profite selbst werden als Residualgröße behandelt. Sie ergeben sich als Überschuss des Werts der produzierten Güter über das, was für Löhne, Grundrenten und den Ausgleich des Kapitalverzehrs erforderlich ist.

Vernachlässigt man die Grundrenten ${ }^{8}$ und unterstellt, dass die „natürlichen“ Preise der produzierten Güter den Arbeitsmengen entsprechen, die für die Produktion erforderlich sind, sei es direkt, bei der Produktion der Güter selbst, sei es auch indirekt, bei der Reproduktion der eingesetzten Kapitalgüter, so entsprechen die Profite dem Überschuss der Arbeitswerte der produzierten Güter über die Löhne. Unterstellt man mit den Klassikern, dass Arbeit die einzige Quelle von Wertschöpfung ist, so fragt sich, warum die Profite überhaupt positiv sind.

Auf diese Frage antwortete Marx: Aufgrund von Ausbeutung! Die Kapitaleigner als gesellschaftliche Klasse nutzen ihre Macht, um sich den bei kapitalistischer Produktion entstehenden Mehrwert anzueignen. In gewissem Sinn ist dies ein spätes Echo des mittelalterlichen Zinsverbots. Wo die Kirche den Zins als Wucher, als Ausnutzung von Notsituationen der Schuldner ansah, sieht Marx die Profite als Ergebnis der Ausnutzung von Macht, durch die der beim Einsatz von Kapital entstehende Mehrwert den Arbeitern vorenthalten wird.

Die Marx'sche Interpretation der Profite als Ergebnis der Ausbeutung erfährt Widerspruch von Ökonom:innen, die der ca. 1870 einsetzenden neoklassischen Analyse verpflichtet sind. Diese wenden die auf dem Ausgleich von Angebot und Nachfrage in den verschiedenen Märkten basierende Preistheorie auf die Märkte für Produktionsfaktoren an, d. h. auf Inputs, und sie interpretieren Zinsen als Entgelte für die Nutzung von „Kapital“. Zu nennen sind insbesondere Böhm-Bawerk, Clark, Wicksteed und Wicksell (Schumpeter, 1954, 1026 ff.). Der US-amerikanische Ökonom John Bates Clark begründete die Vorstellung von einem eigenständigen Produktionsfaktor „Kapital“, der die Essenz der vielfältigen Kapitalgüter ausmacht und als Input in einer volkswirtschaftlichen Produktionsfunktion anzusehen ist. Wicksteed und Wicksell fügten die Beobachtung bei, dass bei konstanten Skalenerträgen in der Produktion und bei einer Bezahlung der Faktoren ent-

8 Nach Ricardo sind Grundrenten Differentialrenten für höherwertiges Land. Das eingesetzte Land niedrigster Qualität erzeugt eine Grundrente von Null. Bei Marx werden Grundrenten als Teil der Profite behandelt 
sprechend ihren Grenzprodukten der gesamte Erlös aus Produktion und Verkauf von Gütern zur Bezahlung der Inputs benötigt wird und es keine Gewinne gibt. Die Aufteilung des Einkommens in Löhne, Grundrenten und Profite bei den Klassikern wird bei den Neoklassikern durch eine Aufteilung in Löhne, Grundrenten und Zinsen ersetzt, wobei für jede Einkommensart das Grenzproduktivitätsprinzip unterstellt wird. Das skizzierte Modell formalisiert genau diese Sicht der Rolle von Kapital und Zinsen.

\section{Folgende Aspekte der Historie sind bemerkenswert:}

- Die Diskussion von Kapitaleinkommen, Zinseinkommen wie Profiten betraf immer auch die Legitimität solcher Einkommen. In Anbetracht dieser Geschichte ist bemerkenswert, wie sehr die heutige Diskussion geprägt ist von der Vorstellung, es gebe geradezu einen Anspruch der Sparenden auf positive Zinseinkommen.

- Das Ausklammern von Geld- und Finanzmärkten bei den englischen Klassikern hat die Analyse wesentlich vereinfacht; es hat aber auch bewirkt, dass die Theorie praktisch nichts über die Märkte zu sagen hat, in denen in der Realität die Zinsen vereinbart werden.

- Das neoklassische Modell ersetzt die Wertgröße „Kapital“ bei den Klassikern durch die Mengengröße „Kapital“ als Input der Produktionsfunktion und die „Profite" der Klassiker durch das Einkommen des Produktionsfaktors „Kapital“ mit dem Zinssatz als Faktorpreis.

Das naive neoklassische Modell mit der Interpretation des Zinses als Preis für die Nutzung von Realkapital dominierte große Teile der Volkswirtschaftslehre vom Beginn des 20. Jahrhunderts bis mindestens in die 1960er Jahre. Vielfach wird es auch heute noch verwendet, da es so schön einfach zu handhaben ist. Jedoch haben die kapitaltheoretischen Kontroversen der 1950er und 1960er Jahre seinen Geltungsanspruch deutlich reduziert.

Den Anstoß gab die englische Ökonomin Joan Robinson (1953). In einem bahnbrechenden Artikel fragte sie, in was für Einheiten „Kapital“ eigentlich gemessen wird. Wie bildet man das Aggregat „Kapital“ aus den vielen verschiedenen Kapitalgütern? Welche Rolle spielen Preise bei dieser Aggregation? Und natürlich: Ist „Kapital“ eine Mengengröße oder eine Wertgröße?

Die nachfolgende Kontroverse konzentrierte sich vor allem auf die Frage, ob bzw. wie man aus den einzelnen Kapitalgütern einen (Mengen)-Index bilden kann und inwiefern die bei der Indexbildung als Gewichte verwandten Preise als Kostenpreise technologisch bestimmt sind (Bliss, 1975). Für die Diskussion hier ist dieses Aggregationspro- blem weniger interessant als die einfache Beobachtung, dass selbst, wenn es nur ein Kapitalgut gibt, dieses aber nicht mit dem Konsumgut übereinstimmt, der Mietpreis für Kapital nicht als Zinssatz interpretiert werden kann und dass im Übrigen der Gleichgewichtszinssatz bzw. die Nettoertragsrate auf Kapital nicht rein technologisch bestimmt werden können. In diesem Punkt haben Robinson und ihre Anhänger eindeutig recht behalten.

Daraus folgte aber nicht notwendigerweise die von Robinson und ihren Anhängern propagierte These, der bei rein technologischer Betrachtung verbleibende Freiheitsgrad werde dadurch ausgefüllt, dass die gesellschaftlichen Machtverhältnisse die Gleichgewichtsprofitrate festlegten, woraus sich dann die Güterpreise als Kostenpreise ergeben. Der Freiheitsgrad wird in einem intertemporalen Marktsystem durch das Verhalten der Nachfrageseite der Volkswirtschaft, Entscheidungen über Konsum und Ersparnis ausgefüllt (Weizsäcker, 1971).

Das Konzept des intertemporalen Marktsystems geht zurück auf Böhm-Bawerk, der deutlich sah, dass ein Gut heute und dasselbe Gut in einem Jahr wirtschaftlich zwei verschiedene Dinge sind. Die moderne Formulierung in der intertemporalen Version des Arrow-Debreu-Modells des allgemeinen Gleichgewichts wurde fast gleichzeitig mit den kapitaltheoretischen Kontroversen entwickelt (Arrow, 1953; auch Debreu, 1959). In diesem Modell erscheinen Zinssätze durchweg als Parameter intertemporaler Austauschverhältnisse, sind aber selbst keine Austauschverhältnisse.

\section{Schlussbemerkung}

Für die Scholastiker war der Zins ein monetäres Phänomen. Die englischen Klassiker dagegen entwickelten eine rein realwirtschaftliche Analyse. Schumpeter bewundert die Radikalität des Ansatzes von Nicholas Barbon, lässt aber offen, ob dessen Idee für die weitere Entwicklung der Wissenschaft nützlich oder schädlich war. Er äußert Bedenken hinsichtlich der Zusammenfassung von Unternehmenserträgen und Zinsen in einer Kategorie, hinsichtlich der Vernachlässigung der Finanzierungsbeziehung und hinsichtlich der Vorstellung, dass der Gläubiger dem Unternehmen nicht eigentlich Geld zur Verfügung stellt, sondern Kapitalgüter, die der Unternehmer mit dem Geld kaufen kann (beinahe wörtlich Adam Smith nach Schumpeter, 1954, 333). Er äußert Unbehagen bezüglich der aus dieser Tradition heraus entstandenen Trennung von realwirtschaftlicher und geldwirtschaftlicher Analyse in der Wirtschaftstheorie.

Wicksell versuchte, diese Trennung zu überwinden, indem er zunächst die realwirtschaftliche Analyse zur De- 
finition eines „natürlichen“ Zinssatzes verwendete und sodann fragte, was passiert, wenn die Institutionen des Geldwesens bewirken, dass der tatsächliche Zins von diesem natürlichen Zinssatz abweicht. Die in der Tradition von Böhm-Bawerk und Wicksell arbeitenden österreichischen und schwedischen Ökonomen (Schumpeter, Mises, Hayek; Åkerman, Lindahl, Myrdal) hatten dann damit zu kämpfen, dass das in einem rein realwirtschaftlichen Modell entwickelte Konzept des „natürlichen“ Zinssatzes in einer Volkswirtschaft, in der das Geldwesen und die Finanzmärkte eine maßgebliche Rolle spielen, nicht ohne weiteres ein Pendant hat. Bei allem Respekt für Wicksell erklärte Gunnar Myrdal (2007) schließlich die beiden Theorieansätze für unvereinbar. Die Annahme, man könne ein Modell, in dem Geld nicht vorkommt, zur Erklärung der Zinssätze in einer monetären Ökonomie verwenden, sei „falsch“ (Myrdal, 2007, 393, kursiv im Original). Ein in sich stimmiges integriertes Modell, das ohne „verrückte“ Annahmen auskäme, ist allerdings noch nicht entwickelt worden (für eine Übersicht über Probleme, die dem entgegenstehen, siehe Hellwig, 1993).

\section{Literatur}

Arrow, K. J. (1953), Le rôle des valeurs boursières pour la répartition la meilleure des risques, Économétrie, Colloques Internationaux du Centre National de la Recherche Scientifique, 11 (1953), 41-47; auf Englisch publiziert als: "The Role of Securities in the Optimal Allocation of Risk Bearing", Review of Economic Studies, 31, 1964, 91-96, Collected Papers, 2, 46-57.

Bliss, C. (1975), Capital Theory and the Distribution of Income.

Böhm-Bawerk, E. v. (1884), Kapital und Kapitalzins, Wagner.
Braudel, F. (1990), Sozialgeschichte des 15.-18. Jahrhunderts, Bd. II: Der Handel, Kindler.

Debreu, G. (1959), Theory of Value: An Axiomatic Analysis of Economic Equilibrium, Cowles Foundation Monograph, 17, Yale University Press.

Hellwig, M. (1993), The Challenge of Monetary Theory, European Economic Review, 37(2-3), 215-242.

Hellwig, M. (2021a), „Die Enteignung der Sparer - eine deutsche Legende“, Frankfurter Allgemeine Zeitung, 2. September.

Hellwig, M. (2021b), Dynamic inefficiency and fiscal interventions in an economy with land and transaction costs, German Economic Review, 22, https://doi.org/10.1515/ger-2020-0110 (13. Oktober 2021).

Hellwig, M. (2021c), Safe Assets, Risky Assets, and Dynamic Inefficiency in Overlapping-Generations Economies, Discussion Paper, 2021/10, Max-Planck-Institut zur Erforschung von Gemeinschaftsgütern.

Homburg, S. (2014), Overaccumulation, Public Debt and the Importance of Land, German Economic Review, 15, 411-435.

Myrdal, G. (2007), Der Gleichgewichtsbegriff als Instrument der geldwirtschaftlichen Analyse, in F. A. Hayek (Hrsg.) (1933), Beiträge zur Geldtheorie, Springer, nachgedruckt bei Springer, 2007.

Robinson, J. (1953), The Production Function and the Theory of Capital, Review of Economic Studies, 21(2), 81-106.

Rothbard, M. (2008), The Mystery of Banking, 2. Aufl., The Ludwig von Mises Institute.

Schumpeter, J. (1954), History of Economic Analysis, Oxford University Press.

Selgin, G. (2012), Those Dishonest Goldsmiths, Financial History Review, 19(3), 269-288.

Sinn, H.-W. (2020), Staatsverschuldung und Dynamische Ineffizienz: Warum der Münchhausen-Trick nicht funktioniert, Wirtschaftsdienst, 100(8), 572-577, https://www.wirtschaftsdienst.eu/inhalt/jahr/2020/heft/8/ beitrag/staatsverschuldung-und-dynamische-ineffizienz-warum-dermuenchhausen-trick-nicht-funktioniert.html (13. Oktober 2021).

Weizsäcker, C. C. v. und H. Krämer (2019), Sparen und Investieren im 21. Jahrhundert: Die große Divergenz, SpringerGabler.

Weizsäcker, C. C. v. (1971), Steady State Capital Theory, Springer Lecture Notes in Operational Research and Mathematical Systems, 54, Springer Verlag.

Wicksell, K. (1898), Geldzins und Güterpreise - Eine Studie über die den Tauschwert des Geldes bestimmenden Ursachen, G. Fischer.

Title: The Interest Rate Should not be Interpreted as a Price

Abstract: The paper criticises interpretations of the interest rate as a price. Prices are exchange ratios for different objects; the interest rate is a parameter characterising an intertemporal exchange ratio but is not itself an exchange ratio. Therefore, negative (real) interest rates can arise naturally without policy interventions. Interpretations of the interest rate as a rental rate for capital are shown to be untenable when there are many goods. The paper also sketches the historical background of the discussion, in particular, the English classical economists and Marx, the neoclassical model of Clarke and Wicksell, and Joan Robinson's criticism of this model.

JEL Classification: B1, D5, E4 